\title{
Primary health care models: learning across continents
}

Contemporary health policies often require new forms of primary care organization for their effective implementation. This has been apparent over the past decade in the UK through the transition from general practice fundholding models to the present NHS (National Health Service) primary care trusts. Equally radical developments in both the context and structures of frontline health service delivery have been evident internationally wherever the tenets of 'Modernization' have held sway. As a result there is now considerable scope for transferable learning between those countries, in every continent, that are now experimenting with novel organizational developments in primary care. Typically these developments are part of attempts to revive not just local health systems but the role of the nation state as well. Although this may seem most obvious in the post dictatorship countries and emergent participatory democracies of Latin America there are also lessons, and fresh opportunities for reciprocal exchange, for practitioners and policy makers in the UK, where recent government guidance has pointed firmly in the direction of more diversity in both primary care provision and ownership (Department of Health, 2006).

Such diversity is a commonplace globally in 'modernizing' health policies through their emphasis on exploiting previously unexplored cross sectoral collaborations and new interprofessional and practice partnerships. Together with innovations in governance, stewardship arrangements for public health and multiple forms of financing these represent a radical agenda for local resource management in today's health systems. Over the past four years our fieldwork has taken us to thirty countries where this framework for policy reforms has applied since 1997 - when a Labour Government took office in the UK promising a 'New NHS' - and led to the identification of six different standard models of primary care (C) 2006 Cambridge University Press organization (Meads, 2005). Each belongs to its particular cultural context. All, however, are also examples of the new corporate organizational developments that together signify the fundamental shift taking place in contemporary primary care, as it moves away from simple forms of uniprofessional partnership towards tomorrow's world of complex stakeholder enterprises.

The new forms of primary care organization correspond broadly with the six administrative regions of the World Health Organization, and the influence of the latter in steering national leaderships is sometimes not too difficult to detect. Across Sub Saharan Africa, for example, in countries such as Uganda and Kenya, the principles of Alma Ata and Health for All are enmeshed in the District Health System model; while the Washington based and impressively autonomous Pan American Health Organization has been positively assertive in its sponsorship of the Community Development Agency version of primary care for such countries as Peru, Costa Rica and Venezuela. In Europe the principle of subsidiarity has ensured more national control over domestic health policy developments. Nevertheless in recent years the Extended General Practice in the UK and elsewhere has grown in tandem with Managed Care organizational changes that have their origins in the USA, and which may now be seen translated into the operation of, for instance, Thailand's competitive Contracting Units for Primary Care and New Zealand's hybrid Primary Health Organizations, with their mix of private and public service professionals.

Alongside the extended general practice, the managed care business, the district health system and the community development agency stands the most surprising of the contemporary organizational developments in primary care: the reformed polyclinic. Its capacity to attract co-payments and private specialist contributions that can even include, in the likes of Bolivia and Belarus, alternative 
medical practitioners and traditional healers, has meant that in countries as far apart as Brazil, Greece and even Australia a revamped version of the old Soviet style cubicled polyclinic has reappeared. In each case its practitioners combine the delivery of specifically targeted interventions, commissioned as part of statutory public health programmes, with their marketing of additional and supplementary private health care services.

The final organizational development in modern primary care is the miscellaneous category of franchised outreach units, invariably in such countries as the Philippines, Japan and Indonesia where there is virtually no history of family medicine. At such locations comprehensive strategies for primary care development are noticeable for their scarcity at any administrative level of government. New organizational developments take place often more by default than by design, with the marketplace the chief determinant of what occupies the frontline of the health care system. With Asian countries the principal hosts to competitive tendering in primary care, it is often private clinics and hospitals that find a commercially advantageous niche, with local charities and international donors to the fore where they do not. Singapore with its government sponsored Singhealth Clinics offers the best global example of a systematic attempt to combine the franchised outreach model with the benefits of the reformed polyclinic.

Here the endpoint is the 'client' or 'customer'. Each organizational type has its own imagery. The 'patient' of the traditional if extended general practice becomes the 'user' of the managed care business, and a 'citizen' in areas where community development agencies assume responsibility for primary care. Across all the six types there are generalizable trends. These include the growing professionalization of community nursing and the move towards population units of 30000 for primary care coverage. It is, however, the differences between the types, and the advantages and drawbacks associated with each, that are more important for both policymakers and professionals to understand.

The significant variation in the roles of the general medical practitioner illustrates this need to align policy and organizational developments in primary care. From clinical care manager in a Scandinavian health centre to secondary care based systems planner and monitor in a South African general hospital; from fee for service income generator in a Canadian physicians' network to an insurance risk assessor in the national Mexican petroleum company, PEMEX: the new diversity of general practitioners highlights the potential for real transformation in future service delivery arrangements of primary care. In seeking to discern which of the new roles might be adapted most productively for the benefit of populations and individuals in the UK, our research suggested that the community development model might have the most to offer. It could both sustain and strengthen the relationships based approach which has always been the outstanding feature of British primary care (Wild et al., 2003). A new publication offers accounts of four international exemplars of each of the six organizational types described briefly above (Meads, 2006), with the Chiclayo MaxSalud Clinic in Central Peru, for instance, illustrating how even prescribing charges and protocols can be more efficiently managed by elected lay committees with their family doctors if the organizational developments are 'fit for purpose' and the local context.

In economically developed countries more health policy is now taught in university business schools than in their medical and nursing counterparts. From the former come the new private sector ideas of 'network', 'learning' and 'virtual' organizations that are exerting an increasingly powerful influence over public health care systems and primary care, in particular. At a time when structural organizational design is the preferred method of policy formulation the research challenge is considerable. In the past the profile of primary care research has largely reflected its major and controlling professional and clinical interests. Its future portfolio clearly needs to embrace the study of the organizational developments, and a current international perspective sheds light on the transformational scale of the contemporary policies that are their source. 


\section{References}

Department of Health. 2006: Our health, our care, our say: a new direction for community services. London: Department of Health Publications.

Meads, G. 2005: Family medicine: future issues and perspectives. In Garcia-Pena, C., Munoz, O., Duran, L. and Vazquez F., editors. Family medicine at the dawn of the 21st century. Mexico: D.F. Instituto Mexicano del Seguro Social, 17-33.

Meads, G. 2006: Primary care in the 21st century. an international perspective. Oxford: Radcliffe Publishing.

Wild, A., Iwami, M. and Meads G. 2003: Different systems, same issues. Primary Care Report 5, 14-19. 